\title{
Development of High Performance Thin Layer Chromatography for Simultaneous Analysis of Lamivudine and Tenofovir Disoproxil Fumarate
}

\author{
Thomas Bizimana ${ }^{1}$, Pierre Claver Kayumba ${ }^{1 *}$, Kagisha Védaste ${ }^{1}$, Kayitare Egide ${ }^{1}$, Eliangiringa Kaale \\ ${ }^{1}$ Department of Pharmacy, School of Medicine and Pharmacy, College of Medicine and Health Sciences, \\ University of Rwanda, P.O. Box 4285, Kigali, Rwanda \\ ${ }^{2}$ Muhimbili University of Health and Allied Sciences, Dar Es Salaam, Tanzania
}

Abstract

\begin{abstract}
Background: The quality of antiretroviral medicines (ARVs) is vital in the management of HIV infection. Nevertheless, the effort for the management of HIV infection is impeded by the increasing problem of counterfeit ARVs. Objectives:The aim of this study was to avail a less expensive, accurate, and precise analytical method for the simultaneous determination of lamivudine and tenofovir disoproxil fumarate in fixed dosage combination. Methods: During the method development and validation, a high performance thin layer chromatography (HPTLC) system with WinCATS software was used. Freshly prepared solutions in methanol were spotted on HPTLC silica gel $60 \mathrm{~F}_{254}$ plates. The mobile phase was composed by $20 \mathrm{~mL}$ of toluene and $10 \mathrm{~mL}$ of methanol, both analytical grades. Results: The retention factor (Rf) was from 0.35 to 0.38 and from 0.57 to 0.59 , respectively for lamivudine and tenofovir disoproxil fumarate. The coefficients of variation (CV) for repeatability were $1.3 \%$ and $3.4 \%$, respectively for tenofovir disoproxil fumarate and lamivudine. The CVs for intermediate precision were 3.9\% and 3.8\%, respectively for lamivudine and tenofovir disoproxil fumarate. The analytical range was $2.000-3.100 \mu \mathrm{g}$ for both lamivudine and tenofovir disoproxil fumarate. Conclusions: This method is recommended in routine analysis of pharmaceutical products containing lamivudine and tenofovir disoproxil fumarate.
\end{abstract}

Keywords: HPTLC, Lamivudine, Tenofovir disoproxil fumarate.

\section{Introduction}

The quality of antiretroviral medicines (ARVs) is vital in the management of HIV infection. Nevertheless, the effort for the management of HIV infection is impeded by the increasing problem of counterfeit ARVs (Nsimba, 2008; WHO, 1999). Because of their importance, their high cost and high rate of consumption, counterfeit copies of ARVs, are likely to be found on the market, especially in developing countries. A strong system of quality control and quality assurance with appropriately validated methods is among the defensive measures to prevent the use of counterfeit ARVs in the local markets (WHO, 1999).

The fixed combination of lamivudine+tenofovir disoproxil fumarate (LT) whose structures are presented in Figure 1 is part of HIV first line treatment (AVERT, 2015) of which the risk of finding counterfeits of this dosage form is high (Nsimba, 2008; WHO, 1999).

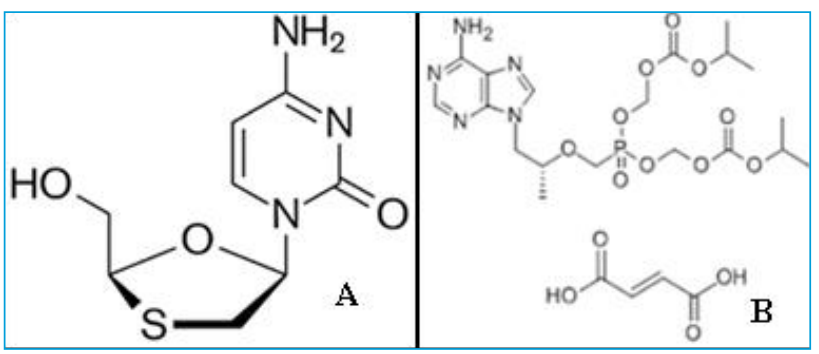

\section{Figure 1. (A) Structure of Lamuvidine, (B) structure of Tenofovir disoproxil fumarate}

The literature shows that the analytical method for this dosage form is High Performance Liquid Chromatography (HPLC) based while it is known that this method is solvent and time consuming during sample preparation and analysis
(Alexander Shikov, Olga Pozharitskaya, Svetlana Ivanova, Valery Makarov, n.d.; Günther \& Schmidt, 2005). A HPTLC method has been developed (Joshi, Nikalje, Shahed, \& Dehghan, 2009) but was using chloroform which is very toxic, especially on the central nervous system (Agency for Toxic Substances and Disease Registry, 1997). Therefore, a HPTLC method using less toxic solvents (methanol and toluene) was needed. The purpose of this study was then to develop and validate a HPTLC method responding to the above mentioned challenges.

\section{Methods}

A method for determination of Lamivudine and Tenofovir disoproxil fumarate in a fixed combination tablet has been developed and validated.

\section{Chemicals and Reagents}

Tablets of lamivudine+tenofovir disoproxil fumarate were obtained from Rwandan National Drug Stores. All solvents (Methanol, toluene, ethyl acetate, acetone, glacial acetic acid) were of analytical grade and were obtained from SIGMA-ALDRICH Company Ltd, (Gillinhan, UK).

\section{Reference standards}

Lamivudine and tenofovir disoproxil fumarate reference standards were obtained from European Directorate for Quality of Medicine and Healthcare (EDQM), (Council of Europe, Strasbourg, France).

\section{Instrumentation}

The instrumentation used was a HPTLC system consisting of Linomat-5 applicator, TLC Scanner 3 and WinCATS software $\vee$ 1.4.3 all from CAMAG, Muttenz Switzerland. 
The samples were spotted with a CAMAG 100-microliter sample syringe (Hamilton, Bonaduz, Switzerland) syringe on silica gel pre-coated glass plate 60 GF254 $(20 \times 10 \mathrm{~cm})$ with $250 \mu \mathrm{m}$ thickness, while the analytical balance used was Adventure ${ }^{\circledR}$ Ohaus Corp. Pine Brook, NJ, USA.

\section{Preparation of solutions}

\section{Preparation of sample solutions}

Twenty tablets were accurately weighed and the average mass per tablet was calculated. The tablets were powdered and the mass of powder equivalent to $50 \mathrm{mg}$ of Lamivudine and $50 \mathrm{mg}$ of Tenofovir disoproxil fumarate was accurately weighed in a $20 \mathrm{~mL}$ volumetric flask. About $15 \mathrm{~mL}$ of methanol was added and to accelerate dissolution, samples were sonicated for about $10 \mathrm{~min}$. The volume was adjusted to $20 \mathrm{~mL}$ using methanol to have stock sample solutions of $2.5 \mathrm{mg} \mathrm{mL}^{-1}$ in Lamivudine and $2.5 \mathrm{mg} \mathrm{mL}^{-1}$ in Tenofovir disoproxil fumarate.

\section{Preparation of reference standard solutions}

The standard stock solutions of $2.5 \mathrm{mg} / \mathrm{ml}$ lamivudine and $2.5 \mathrm{mg} / \mathrm{ml}$ tenofovir disoproxil fumarate were prepared as follows: $50 \mathrm{mg}$ of Lamivudine and $50 \mathrm{mg}$ of tenofovir disoproxil fumarate were accurately weighed in a $20 \mathrm{ml}$ volumetric flask. About $15 \mathrm{~mL}$ of methanol were added and sonicated for $10 \mathrm{~min}$ to accelerate dissolution. The volume was adjusted to $20 \mathrm{~mL}$ using the same solvent.

\section{Method development}

The HPTLC method was performed on $20 \mathrm{~cm} \times 10 \mathrm{~cm}$ HPTLC glass plates coated with $200-\mu m$ layer thickness of silica gel 60F 254 (E. Merck, Germany). Samples were applied as $8 \mathrm{~mm}$ width bands using Camag 100 microlitre sample syringe (Hamilton, Switzerland) with a Camag Linomat 5 applicator (Camag, Switzerland). A constant application rate of $5 \mu \mathrm{L}^{\mathrm{s}-1}$ was used and solutions were applied to the plate as $8 \mathrm{~mm}$ bands at a distance of $15 \mathrm{~mm}$ from the edge of the plate. Linear ascending development at a distance of $7 \mathrm{~cm}$ with different mobile phases were carried out in a twin trough glass chamber (Camag) $(20 \times 10$ $\mathrm{cm})$ previously saturated with mobile phase vapour for 20 min at room temperature $\left(25 \pm 2^{\circ} \mathrm{C}\right)$.

After development, plates were air-dried for about 10 minutes. Scanning was performed using Camag TLC scanner 3 in the absorbance mode and operated by winCATS software (version 1.4.3). The source of radiation was a deuterium lamp emitting a continuous UV spectrum in the range $200-800 \mathrm{~nm}$. The slit dimensions were $5 \mathrm{~mm}$ $\times 0.45 \mathrm{~mm}$ and the scanning speed was $100 \mathrm{~mm} / \mathrm{s}$.

\section{Method validation}

\section{Specificity or selectivity}

In our case, evaluation of the effect of the matrix and dissolving solvent was concerned. We have evaluated whether responses were not influenced by the matrix or the dissolving solvent. A placebo solution was prepared according to the composition of LT tablet, and a blank/ diluent was prepared. Both the placebo solution and the blank were spotted three times during each chromatographic run and the chromatogram was analyzed.

\section{Linearity and Range}

We studied the linearity of the candidate method by evaluating the correlation coefficient (R2), the slope, the intercept with the confidence level and a graphical representation (regression model) (ICH, 1996), (Chung Chow Chan, Herman Lam, 2010). From a stock solution of a mixture containing lamivudine and tenofovir disoproxil fumarate in methanol, five standard solution levels of the analytes at 80\%, 90\%, 100\%, 110\% and 120\% were prepared using serial dilutions. Three spots were made at each concentration level, adequately bracketed by the standard. Samples were spotted from the lowest concentration to the highest concentration to reduce the effects, if any, of carryover from the higher concentration samples. The spotted plate was developed and scanned to get the densitograms. Using peak areas, a calibration plot of peak areas vs. concentration was made. The procedure was repeated for three consecutive days.

\section{Precision/Repeatability (Intra-day precision)}

We chose to use six replicates of the sample preparation at $100 \%$, where each solution was spotted three times (Chung Chow Chan, Herman Lam, 2010). Then, the mean and recovery percentage were calculated and results were presented in a table. The percentage relative standard deviation of the assay or recovery values were evaluated (Chung Chow Chan, Herman Lam, 2010).

\section{Accuracy/recovery}

\section{Inter-day Accuracy}

Three different sample stock solutions were prepared and from each stock solution, three levels that correspond to $80 \%, 100 \%$ and $120 \%$ of the nominal concentration were prepared (Chung Chow Chan, Herman Lam, 2010). Then three determinations were performed at all three levels over three consecutive days and the chromatograms were evaluated where peak areas were recorded in a table.

\section{Intra-day accuracy}

The same procedure as for inter-day accuracy was used; all steps were performed three times by the same person and on the same day.

\section{Results}

\section{Method development}

\section{Selection of suitable mobile phase and saturation time}

A mobile phase composed by $20 \mathrm{ml}$ of toluene and $10 \mathrm{ml}$ of methanol, both analytical grades at room temperature (25 \pm $2{ }^{\circ} \mathrm{C}$ ) was found the most appropriate for the separation of lamivudine and tenofovir disoproxil fumarate in a solution of methanol. This mobile phase was maintained based on safety of solvents, resolution and sharpness of peaks. Different saturation times from 15 to 30 minutes were tried. After the comparison of all data, it was found that the saturation time of 20 minutes gave a better resolution of lamivudine and tenofovir disoproxil fumarate peaks at RF values of $0.38 \pm 0.01$ and $0.57 \pm 0.01$ respectively, without inference with the solvent front and the matrix as shown on densitogram. 
Selection of the optimum wavelength in simultaneous determination of lamivudine and tenofovir disoproxil fumarate

In simultaneous determination of lamivudine and tenofovir disoproxil fumarate, a wavelength $\lambda=270 \mathrm{~nm}$ was shown to be the optimum scanning wavelength (Figure 2).

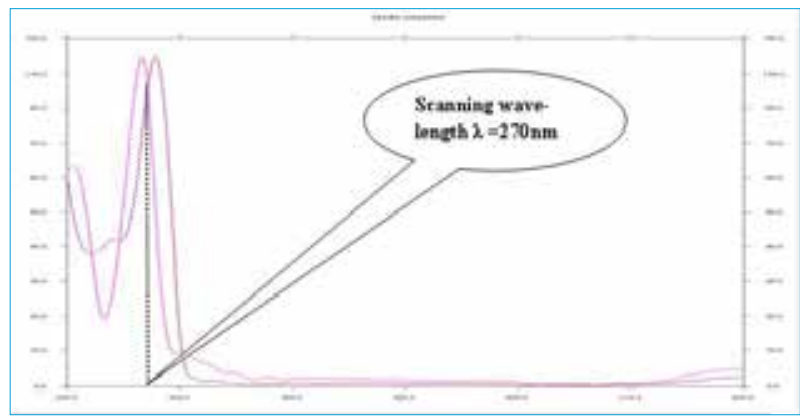

Figure 2. Overlaid full spectra UV-VIS of lamivudine and tenofovir disoproxil fumarate scanned between 200 and $800 \mathrm{~nm}$, showing the maximum coabsorption at $270 \mathrm{~nm}$

\section{System suitability}

After varying temperature and humidity, best results of resolution were obtained when the room was cooled between 18 and $20^{\circ} \mathrm{C}$ with a relative humidity varying between $40 \%$ and $60 \%$. Under these conditions, the system was suitable with a good resolution as will be shown in Figure 3 on specificity of results.

Response function: polynomial regression model for lamivudine and tenofovir disoproxil fumarate

According to literature review, the polynomial regression model is more suitable than other regression models in case of HPTLC (John H. Mcdonald, 2014; Kaale et al., 2013). Table 1 shows the values of different parameters of the regression function for both lamivudine and tenofovir disoproxil fumarate.

Table 1. Summary of response function of lamivudine and tenofovir disoproxil fumarate

\begin{tabular}{|c|c|c|c|c|}
\hline $\begin{array}{l}\text { Response } \\
\text { function } \\
(k=5, m=3 \text {, } \\
n=3)\end{array}$ & Day 1 & Day 2 & Day 3 & $\begin{array}{l}\text { Average } \\
\text { of } 3 \text { days }\end{array}$ \\
\hline \multicolumn{5}{|l|}{ Lamivudine } \\
\hline $\begin{array}{l}\text { Polynomial } \\
\text { term }\end{array}$ & -1355.200 & -195.330 & -2023.300 & -1184.900 \\
\hline Slope & 10252.000 & 3983.600 & 14180.000 & 9436.500 \\
\hline Intercept & -1022.800 & 6716.200 & -6245.900 & -136.050 \\
\hline $\mathrm{R}^{2}$ & 0.9940 & 0.9950 & 0.9995 & 0.9974 \\
\hline \multicolumn{5}{|c|}{ Tenofovir disoproxil fumarate } \\
\hline $\begin{array}{l}\text { Polynomial } \\
\text { term }\end{array}$ & -1035.000 & 404.850 & -1071.800 & -576.390 \\
\hline Slope & 7306.000 & -218.900 & 7415.000 & 4860.800 \\
\hline Intercept & -5153.600 & 4686.600 & -4637.700 & -1709.300 \\
\hline $\mathrm{R}^{2}$ & 0.9995 & 0.9967 & 0.9944 & 0.9979 \\
\hline
\end{tabular}

From day 1 to day 3 for lamivudine and tenofovir disoproxil fumarate, the response function was good with R2 greater than 0.98 .

\section{Method validation}

\section{Specificity results}

No peaks were attributable to the blank/diluent or placebo (Figure 3).

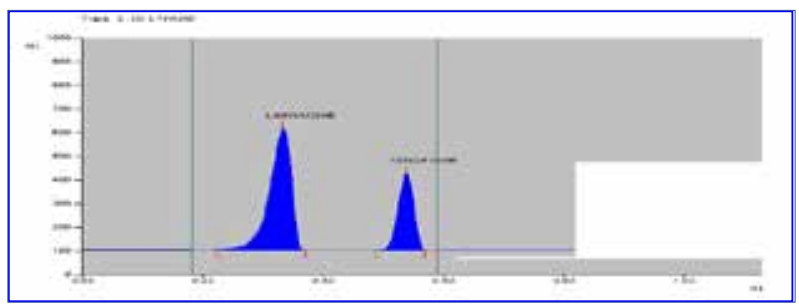

Figure 3. Typical densitograms of a solution containing Lamivudine and Tenofovir disoproxil fumarate

Using a mobile phase composed by Toluene-methanol (20:10), saturation time of $20 \mathrm{~min}$ on a $20 \mathrm{~cm} \times 10 \mathrm{~cm}$ TLC plate and application volume of $5 \mu \mathrm{L}$ at a detection wave length of $270 \mathrm{~nm}$, the Retention factor (Rf) values are 0.37 0.57 respectively for lamivudine and Tenofovir disoproxil fumarate.

The candidate method has been qualified specific, since no peak has been detected from the dissolving solvent and blank placebo. This shows that the method is selective and specific to lamivudine and tenofovir disoproxil fumarate.

\section{Linearity/Range}

Linearity results for Lamivudine and Tenofovir disoproxil fumarateare presented in Table 2.

Table 2. Lamivudine and Tenofovir disoproxil fumarate linear function $(m=5 ; n=3)$

\begin{tabular}{lll}
\hline & \multicolumn{2}{c}{ Value } \\
Criteria & Lamivudine & Tenofovir disoproxil fumarate \\
\hline Range (ng) & $2008-3018$ & $2004-3066$ \\
Slope & 3484.8 & 1950.2 \\
Intercept & 7188.4 & 1891.6 \\
$\mathbf{r}^{2}$ & 0.9873 & 0.9903 \\
\hline
\end{tabular}

The linear function of the studied method is good since $r^{2}$ is greater than 0.98 for both lamivudine and tenofovir disoproxil fumarate. There is correlation between detected peak area and amount of the substance present in the sample.

\section{Precision}

The coefficient of variation (CV) found after calculation of recovered amount was less than $2 \%$ for tenofovir disoproxil fumarate but greater than $2 \%$ for lamivudine. In terms of repeatability, the method candidate is more precise for tenofovir disoproxil fumarate than for lamivudine. The intermediate precision as expressed in terms of the 
percentage relative standard deviation (\%rsd) was $2.3 \%$ for lamivudine and $2.4 \%$ for tenofovir disoproxil fumarate. This means that when the operator changes the candidate method is more precise for lamivudine than for tenofovir disoproxil fumarate. However, a good method should have a \%rsd that is less than 2.0\% (Chung Chow Chan, Herman Lam, 2010).

\section{Accuracy}

Using the calibration curve, we have determined the percentage recovery for a sample solution. Standard solutions as controls were spotted in triplicate at 80, 100 and 120\% (Table 3).

Table 3. Accuracy results for lamivudine $(m=3 ; k=3 ; n=3)$

\begin{tabular}{|c|c|c|c|c|c|c|c|}
\hline \multirow[t]{2}{*}{ Sample } & \multirow[t]{2}{*}{$\mathrm{Rf}$} & \multirow{2}{*}{$\begin{array}{l}\text { Amount injected } \\
\text { in } \mu \mathrm{g}\end{array}$} & \multirow{2}{*}{$\begin{array}{l}\text { Amountrecovered } \\
\text { in } \mu \mathrm{g}\end{array}$} & \multicolumn{2}{|c|}{$\beta$-Expectation limits } & \multirow{2}{*}{$\begin{array}{l}\text { Accuracy Level } \\
85 \%-115 \%\end{array}$} & \multirow{2}{*}{$\begin{array}{l}\text { Average Accuracy level } \\
85 \%-115 \%\end{array}$} \\
\hline & & & & $85 \%$ & $115 \%$ & & \\
\hline 80\%U1 & 0.39 & 2.012 & 1.9796 & 1.7102 & 2.3138 & 98.390 & \\
\hline 80\%U2 & 0.38 & 2.012 & 2.1781 & 1.7102 & 2.3138 & 108.255 & 106.284 \\
\hline $80 \%$ U3 & 0.38 & 2.012 & 2.2576 & 1.7102 & 2.3138 & 112.207 & \\
\hline 100\%U1 & 0.39 & 2.515 & 2.5745 & 2.13775 & 2.89225 & 102.366 & \\
\hline 100\%U2 & 0.38 & 2.515 & 2.7682 & 2.13775 & 2.89225 & 110.068 & 107.512 \\
\hline 100\%U3 & 0.38 & 2.515 & 2.7691 & 2.13775 & 2.89225 & 110.103 & \\
\hline 120\%U1 & 0.38 & 3.018 & 3.1597 & 2.5653 & 3.4707 & 104.695 & \\
\hline $120 \% \cup 2$ & 0.38 & 3.018 & 3.5776 & 2.5653 & 3.4707 & 118.542 & 113.940 \\
\hline $120 \% \cup 3$ & 0.38 & 3.018 & 3.5788 & 2.5653 & 3.4707 & 118.582 & \\
\hline
\end{tabular}

This method is accurate for lamivudine since the average recovery as shown in the Table 3 was always within the $\beta$ - expectation limits (Table 4).

Table 4. Accuracy results for tenofovir disoproxil fumarate

\begin{tabular}{|c|c|c|c|c|c|c|c|}
\hline \multirow[b]{2}{*}{ Sample } & \multirow[b]{2}{*}{$\mathrm{Rf}$} & \multirow{2}{*}{$\begin{array}{l}\text { Amount Injected } \\
\text { in } \mu \mathrm{g}\end{array}$} & \multirow{2}{*}{$\begin{array}{l}\text { Amount recovered } \\
\text { in } \mu \mathrm{g}\end{array}$} & \multicolumn{2}{|c|}{$\beta$-Expectation limits } & \multirow{2}{*}{$\begin{array}{l}\text { Accuracy level } \\
85 \%-115 \%\end{array}$} & \multirow{2}{*}{$\begin{array}{l}\text { Average Accuracy leve } \\
85 \%-115 \%\end{array}$} \\
\hline & & & & $85 \%$ & $115 \%$ & & \\
\hline $80 \% \cup 1$ & 0.59 & 2.016 & 2.009 & 1.7136 & 2.3184 & 99.653 & \\
\hline $80 \% \cup 2$ & 0.57 & 2.016 & 2.056 & 1.7136 & 2.3184 & 101.984 & 104.282 \\
\hline $80 \% \cup 3$ & 0.57 & 2.016 & 2.242 & 1.7136 & 2.3184 & 111.210 & \\
\hline 100\%U1 & 0.58 & 2.520 & 2.376 & 2.142 & 2.898 & 94.286 & \\
\hline $100 \%$ U2 & 0.57 & 2.520 & 2.518 & 2.142 & 2.898 & 99.921 & 97.712 \\
\hline $100 \%$ U3 & 0.57 & 2.520 & 2.493 & 2.142 & 2.898 & 98.929 & \\
\hline $120 \%$ U1 & 0.57 & 3.024 & 2.736 & 2.5704 & 3.4776 & 90.476 & \\
\hline $120 \%$ U2 & 0.57 & 3.024 & 2.969 & 2.5704 & 3.4776 & 98.181 & 96.186 \\
\hline $120 \% \cup 3$ & 0.57 & 3.024 & 3.021 & 2.5704 & 3.4776 & 99.901 & \\
\hline
\end{tabular}

This method is also accurate for tenofovir disoproxil fumarate since the average recovery was always within the $\beta$ - expectation limits.

\section{Discussion}

This method was developed and validated following guidelines of the International Conference on Harmonization-Text on Validation of Analytical Procedures (ICH, 1996). Results of this research shows suitability of parameters of an analytical assay method. It does not fit for stability analysis. It has good specificity, linearity, precision and accuracy. The recovery percentage did not exceed the acceptance interval of 85-110\%, for lamivudine and tenofovir disoproxil fumarate. The retention factors from sample solutions were the same as the retention factors from standard solutions spiked in the matrix and there was no interference of the matrix. This method does not show any peaks from the solvent. It presents advantages compared to High Performance Liquid Chromatography, by the fact that it produces analytical results of good quality and consumes less time and solvents. It is adapted to the working conditions in the East African Region, where the existing methods were not giving good results (Esters et al., 2006).

\section{Conclusion}

A simple, rapid, cheap, specific, precise and accurate HPTLC method for the simultaneous determination of lamivudine and tenofovir disoproxil fumarate has been developed and validated. Its repeatability, accuracy and selectivity are shown to be good and the mobile phase is composed by solvents that are not very toxic. This method is recommended for routine simultaneous determination of lamivudine and tenofovir disoproxil fumarate. 


\section{Study limitations}

During method development and validation, we assumed that extraction was $100 \%$, while it might have been lower due to the variation of working conditions (temperature and humidity) that can affect the solubility of lamivudine and tenofovir disoproxil fumarate.

\section{Acknowledgements}

This work was supported by "Training Health Researchers into Vocational Excellence in East Africa (THRiVE)", grant number 087540 funded by the Welcome Trust. Its contents are solely the responsibility of the authors and do not necessarily represent the official views of the supporting offices. We also acknowledge Muhimbili University of Health and Allied Sciences (MUHAS) for having hosted this research and researchers, and the University of Rwanda/ former National University of Rwanda, for having accorded a study leave to researchers.

\section{Conflict of interests}

None

\section{Authors' contributions}

BT: Main researcher/Author; KPC: Coordinator of activities in Rwanda and text proofreader; KV: Sampling, reagents procurement and advisor on laboratory activities; KE: Administrative issues (authorizations, funds requisition, and follow-up), statistical analysis and texts proofreading; EK: Laboratory activities coordinator in Tanzania and supervisor

\section{References}

Agency for Toxic Substances and Disease Registry. (1997). TOXICOLOGICAL PROFILE FOR CHLOROFORM

Alexander Shikov*, Olga Pozharitskaya, Svetlana Ivanova, Valery Makarov, D. V. K. (n.d.). A comparison between HPLC and HPTLC for the separation and quantification of boswellic acids in Boswellia serrata extracts. CAMAG-Planar Chromatography in Practice. Muttenz (Switzerland).

AVERT. (2015). Universal Access to HIV Treatment I AVERT Universal Access to HIV Treatment Universal Access to HIV Treatment | AVERT. AVERTing VIV and AIDS, 1-9.

Chung Chow Chan, Herman Lam, X. M. Z. (2010). Practical approaches to method validation and essential instrument qualification. New Jersey: John Willey \& Sons, Inc.

Esters, V., Angenot, L., Brandt, V., Frédérich, M., Tits,

M., Van Nerum, C., Hubert, P. (2006). Validation of a highperformance thin-layer chromatography/densitometry method for the quantitative determination of glucosamine in a herbal dietary supplement. Journal of Chromatography. A, 1112(1-2), 156-64. http://doi. org/10.1016/j.chroma.2006.01.035

Günther, M., \& Schmidt, P. C. (2005). Comparison between HPLC and HPTLC-densitometry for the determination of harpagoside from Harpagophytum procumbens $\mathrm{CO}(2)$-extracts. Journal of Pharmaceutical and Biomedical Analysis, 37(4), 817-21. http://doi. org/10.1016/j.jpba.2004.11.037
ICH. (1996). Guidance for Industry Q2B Validation of Analytical Procedures: Methodology Guidance for Industry Q2B Validation of Analytical Procedures: Methodology.

JOHN H. MCDONALD. (2014). HANDBOOK OF BIOLOLOGICAL STATISTICS (THIRD). Baltimore, Maryland, U.S.A.: SPARKY HOUSE PUBLISHING. http:// doi.org/10.1007/s13398-014-0173-7.2

Joshi, M., Nikalje, a P., Shahed, M., \& Dehghan, M. (2009). HPTLC Method for the Simultaneous Estimation of Emtricitabine and Tenofovir in Tablet Dosage Form. Indian Journal of Pharmaceutical Sciences, 71(1), 95-7. http://doi.org/10.4103/0250$474 \times .51951$

Kaale, E., Nyamweru, B. C., Manyanga, V., Chambuso, M., \& Layloff, T. (2013). The development and validation of a Thin Layer Chromatography densitometry method for the analysis of diclofenac sodium tablets. International Journal of Chemical and Analytical Science, 4(2), 73-79. http://doi.org/10.1016/j. ijcas.2013.05.001

Nsimba, S. E. D. (2008). PROBLEMS ASSOCIATED WITH SUBSTANDARD AND COUNTERFEIT DRUGS IN DEVELOPING COUNTRIES: A REVIEW ARTICLE ON GLOBAL IMPLICATIONS OF COUNTERFEIT DRUGS IN THE ERA OF ANTI-RETROVIRAL (ARVS) DRUGS IN A FREE MARKET ECONOMY. East African Journal of Public Health, 5(3), 205-210.

WHO. (1999). WHO EDM OSM 99.1 counterfeit guidelines.pdf. Geneva: WHO. Retrieved from http://whalibdoc. who.int/ha/1999/WHO_EDM QSM_99.1.pdf 